\title{
Pain Management Strategies Postoperatively in Arthroscopy of Hip: A review Article
}

\author{
Collin LaPorte ${ }^{1,2 *}$, Michael D Rahl ${ }^{2}$, Olufemi R Ayeni ${ }^{3}$ and Travis J Menge ${ }^{1,2}$ \\ ${ }^{1}$ Spectrum Health Medical Group Orthopedics \& Sports Medicine, Grand Rapids, USA \\ ${ }^{2}$ Michigan State University College of Human Medicine, Grand Rapids, USA \\ ${ }^{3}$ Division of Orthopedic Surgery, McMaster University, Canada
}

*Corresponding author: Collin LaPorte, Spectrum Health Medical Group Orthopedics \& Sports Medicine, Grand Rapids, USA.

\begin{abstract}
Hip arthroscopy is an increasingly rapid field in the treatment of multiple hip conditions, owing to its important diagnostic and therapeutic benefit. As these patients lack a consistent pain relief plan, effective post-operative pain control appears to be a concern. Several methods were used to identify a method that decreases post-operative pain, narcotic intake and hospital and treatment system costs. This article aims to study and report the relevant findings of the previous paper "Post-operative pain management strategies in hip arthroscopy."

Latest research encourages the use of a multimodal approach to the treatment of postoperative pain in hip arthroscopic patients. In tandem with peripheral nerve blocks or intraoperative anesthetic injection a pre- and after-operative analgesic regimen is used, patients experience lower discomfort and post-operative narcotic use. Different methods are similar in post-operative pain and opioid use. However, of those undergoing intraarticular (IA) or Local Anesthetic Infiltration (LAI), postoperative risks relative to peripheral nervous blocks are smaller.

Latest trials have demonstrated that the best and most reliable, multi-modal treatment for the reduction of postoperative pain in these patients may be intraoperative techniques such as IA injection or LAI in combination with a pre and postoperative analgesy. Furthermore, failure to use the peripheral nerve block can result in lower anesthesia procedural fees and operating room turnover, thereby lowering patients' costs and increasing facility effectiveness.
\end{abstract}

\section{Introduction}

The Hip arthroscopy is gaining in popularity among the orthopedic surgeons because of its important diagnostic and therapeutic importance in the treatment of common hip disorders such as Femoroacetabular impingement (FAI). While the prevalence of hip arthroscopy for the treatment of hip disease has improved lately, post-operative pain relief appears to be a formidable task for orthopedic surgeons. There is a lack of consistent guidelines for hip arthroscopy postoperative pain relief, possibly because of the lack of high-quality comparative trials investigating the feasibility of multiple therapies.

The aim of PPMS in Hip Arthroscopy [1] was to provide a detailed, up-to-date analysis. Present postoperative pain control literature Hip arthroscopy procedures in patients. It also provides a source for orthopedic surgeons to decide which procedure, or variation thereof, in hip arthroscopy is appropriate for their practice and patients. This statement is intended in advance to review the most relevant conclusions of Hip Arthroscopy Postoperative Pain Relief Strategies [1] and to focus on the potential effects of this paper.

\section{Important Conclusions}

Several new experiments have shown the efficacy of oral drugs for pain relief in the patients who undergo severe orthopedic surgery, such as acetaminophen, gabapentin, and cyclobenzaprine [2-4]. However, these medications have not been tested thoroughly 
in hip arthroscopy patients. On the opposite, Celecoxib has been extensively tested in hip arthroscopies and has demonstrated its high oral bioavailability, fast absorption, and selective cyclooxygenase (COX)-2 inhibition to make it an effective oral antiinflammatorium (NSAID) [5]. Preoperative celecoxib resulted in slightly lower pain scale (VAS) of 1,12 and 24 hours postoperatively compared with placebo [5,6] in two randomized controlled trials. In comparison, patients getting celecoxib spent less time in the PACU than in placebo $[5,6]$.

There has been growing interest in treating the postoperative pain of hip arthroscopic patients with the use of peripheral nerve blocks, such as Lumbar Plexus Block (LPB), Femoral Nerve Block (FNB) and Fascia Iliaca Block (FIB). The VAS pain values in PACU are statistically, though not clinically substantially lower than that of general anesthesia alone, or placebo $[7,8]$ for patients suffering from LPB. However, patients with LPB required fewer postoperative narcotic, anti-emetic and ketorolactic drugs than the control group [7]. In patients receiving FMB, intraoperative and postoperative opioids are usually less necessary than in patients receiving general anesthesia alone or placebo $[9,10]$. In patients receiving FNB pain values were lower than in general anesthesia alone, but in the PACU period was higher in patients receiving FNB [10]. Importantly, patient satisfaction was higher, and the discharge period was shorter in FNB patients compared with pain IV morphine [11] patients. In PACU, FIB patients were reasonably successful (3.85/10) [12] during hip arthroscopy.

Although peripheral nerve blocks can alleviate postoperative pain effectively, they are not complicated. Intravascular injection, iatrogenic nerve trauma, postoperative slips, inflammation and rebound pain following discharge are risk for peripheral nerve blocks [13-15]. Furthermore, peripheral nerve blocks require specialist instruments and an anesthesiologist to operate them and raise patient and hospital costs.

Local intra-articular (IA) anesthetic injections have proved similarly successful relative to FNB. Childs S, et al. [14], recorded no major difference between FNB and pain scores for 1, 3 and 6 weeks. Importantly, postoperative drops were slightly smaller in the IA injection community ( 5 versus $19, \mathrm{p}<0,001$ ). In the IA injection group, there was also a lower prevalence of postoperative peripheral neuritis relative to the FNB group ( 2 vs $26 \mathrm{p}<0.001$ ). IA injection of local anesthetics thus offers a beneficial alternative to FNB for post-operative pain relief, since the treatment is greatly minimized, and the pain values are identical to FNB. In PACU, preoperative celecoxib with acetaminophen and IA injection with morphine and clonidine is greatly reduced as compared to patients who administered oral analgesics alone. However, there were comparable pain ratings between the two classes and no major gap in time for release [16]. Thus, clonidine and morphine IA injection can decrease complications associated with postoperative opioid use, such as respiratory distress and dependence, while providing sufficient analgesia to hip arthroscopy patients.
Local anesthesia penetration (LAI) has increasingly become an important option for orthopedic surgeons to costly operations, such as peripheral nerve blocks. In a research carried out by Philippi MT, et al. [17], intraoperative LAI patients called for fewer postoperative femoral nerve blocks in comparison to the non-LAI community. However, no substantial difference was found between the PACU classes in opioid intake $(p=0.740)$ [17]. In contrast to FIB, patients getting LAI had less discomfort after surgery. Furthermore, the total intake of morphine in the LAI group was twice as low, resulting in less nausea and vomiting than that of the FIB group 24 hours later [18]. Of note, patients receiving LAI required considerably more rescue medicines in contrast to LAI injection $(2,33 \mathrm{mg}$ vs. $0,57 \mathrm{mg}, \mathrm{p}$ $=0,036)$. However, VAS pain ratings did not vary statistically among the groups at 1 to 2 hours after surgery. Thus, LAI is a successful treatment with comparable pain relief effects to peripheral nerve blocks, without intravascular injection risks, iatrogenic nerve disruption, postoperative slips and higher patient and hospital costs. The surgeon performs LAI intraoperatively and does not require ultrasound advice that will decrease the turnover of the operating room time.

\section{Implications}

The reports in recent literature endorse the use of a multimodal approach to postoperative pain control in hip arthroscopy patients. When a multi-modal strategy is employed that is a pre- and postoperative analgesic regime, in tandem with peripheral nerve block or intraoperative anesthetic injection, patients have less pain and postoperative narcotic ingestion. Different methods are similar in post-operative pain and opioid use. However, post-operative symptoms are fewer compared to peripheral nerve blocks in those undergoing IA or LAI. Furthermore, peripheral nerve blocks can lead to intravascular injection, iatrogenic nervous injury and involve highly qualified anesthesiologists which result in increased costs associated with the procedure. IA injection and LAI are quick procedures by the orthopedic surgeon done intraoperatively. Using these intraoperative procedures may improve operating room performance and lower costs for the patient and the hospital by minimizing recovery times and preventing procedural anesthesia charges. A multimodal solution consisting of pre-and after-surgical analgesics paired with IA injection or LAI may thus be the best technique for treating post-operative pain and increasing the costeffectiveness of hip arthroscopy.

\section{Acknowledgement}

None.

\section{Conflict of Interest}

No conflicts of interest.

\section{References}

1. LaPorte C, Rahl MD, Ayeni OR, Travis J Menge (2019) Postoperative Pain Management Strategies in Hip Arthroscopy. Curr Rev Musculoskelet Med 12(4): 479-485. 
2. Schug SA, Sidebotham DA, Mc Guinnety M, Thomas J, Fox L (1998) Acetaminophen as an Adjunct to Morphine by Patient Controlled Analgesia in the Management of Acute Postoperative Pain. Anesth Analg 87(2): 368-372.

3. Han C, Li X, Jiang H, Jian Xiong Ma, Xin Long Ma (2016) The use of gabapentin in the management of postoperative pain after total hip arthroplasty: a meta-analysis of randomised controlled trials. J Orthop Surg Res 11(1): 79.

4. Witenko C, Moorman Li R, Motycka C, Kevin Duane, Juan Hincapie Castillo, et al. (2014) Considerations for the appropriate use of skeletal muscle relaxants for the management of acute low back pain. P T 39(6): 427-435.

5. Kahlenberg CA, Patel RM, Knesek M, Vehniah K Tjong, Kevin Sonn, et al. (2017) Efficacy of Celecoxib for Early Postoperative Pain Management in Hip Arthroscopy: A Prospective Randomized Placebo-Controlled Study. Arthroscopy 33(6): 1180-1185.

6. Zhang Z, Zhang Z, Zhu W, Yaqing Du (2014) Efficacy of celecoxib for pain management after arthroscopic surgery of hip: a prospective randomized placebo-controlled study. Eur J Orthop Surg Traumatol 24(6): 919-923.

7. Schroeder KM, Donnelly MJ, Anderson BM, Michael P Ford, James S Keene (2013) The Analgesic Impact of Preoperative Lumbar Plexus Blocks for Hip Arthroscopy. A Retrospective Review. HIP Int 23(1): 93-98.

8. Ya Deau JT, Tedore T, Goytizolo EA, David H Kim, Douglas S T Green, et al. (2012) Lumbar plexus blockade reduces pain after hip arthroscopy: a prospective randomized controlled trial. Anesth Analg 115(4): 968-972.

9. Dold AP, Murnaghan L, Xing J, Faraj W Abdallah, Richard Brul, et al. (2014) Preoperative Femoral Nerve Block in Hip Arthroscopic Surgery: A Retrospective Review of 108 Consecutive Cases. Am J Sports Med 42(1): 144-149.

10. Xing JG, Abdallah FW, Brull R, Stephanie Oldfield, Andrew Dold, et al. (2015) Preoperative Femoral Nerve Block for Hip Arthroscopy: A Randomized, Triple Masked Controlled Trial. Am J Sports Med 43(11): 2680-2687.
11. Ward JP, Albert DB, Altman R, Rachel Y Goldstein, Germaine Cuff, et al. (2012) Are Femoral Nerve Blocks Effective for Early Postoperative Pain Management After Hip Arthroscopy? Arthroscopy 28(8): 1064-1069.

12. Purcell RL, Nappo KE, Griffin DW, Michael Mc Cabe, Terrence Anderson, et al. (2018) Fascia iliaca blockade with the addition of liposomal bupivacaine vs. plain bupivacaine for perioperative pain management following hip arthroscopy. Knee Surg Sports Traumatol Arthrosc 26(8): 2536-2541.

13. Stein BE, Srikumaran U, Tan EW, Michael T Freehill, John H Wilckens (2012) Lower-Extremity Peripheral Nerve Blocks in the Perioperative Pain Management of Orthopaedic Patients. J Bone Joint Surg Am 94(22): e167.

14. Childs S, Pyne S, Nandra K, Wajeeh Bakhsh, Atif Mustafa, et al. (2017) The Effect of Intra-articular Cocktail Versus Femoral Nerve Block for Patients Undergoing Hip Arthroscopy. Arthroscopy 33(12): 2170-2176.

15. Louw A, Diener I, Butler DS, Emilio J Puentedura (2013) Preoperative Education Addressing Postoperative Pain in Total Joint Arthroplasty: Review of Content and Educational Delivery Methods. Physiother Theory Pract 29(3): 175-194.

16. Cogan CJ, Knesek M, Tjong VK, Rueben Nair, Cynthia Kahlenberg, et al. (2016) Assessment of Intraoperative Intra-articular Morphine and Clonidine Injection in the Acute Postoperative Period After Hip Arthroscopy. Orthop J Sports Med 4(2): 2325967116631335.

17. Philippi MT, Kahn TL, Adeyemi TF, Travis G Maak, Stephen K Aoki (2018) Extracapsular local infiltration analgesia in hip arthroscopy: a retrospective study. J Hip Preserv Surg 5(1): 60-65.

18. Garner M, Alsheemeri Z, Sardesai, Vikas Khanduja (2016) A Prospective Randomized Controlled Trial Comparing the Efficacy of Fascia Iliaca Compartment Block Versus Local Anesthetic Infiltration After Hip Arthroscopic Surgery. Arthroscopy 33(1): 125-132. 